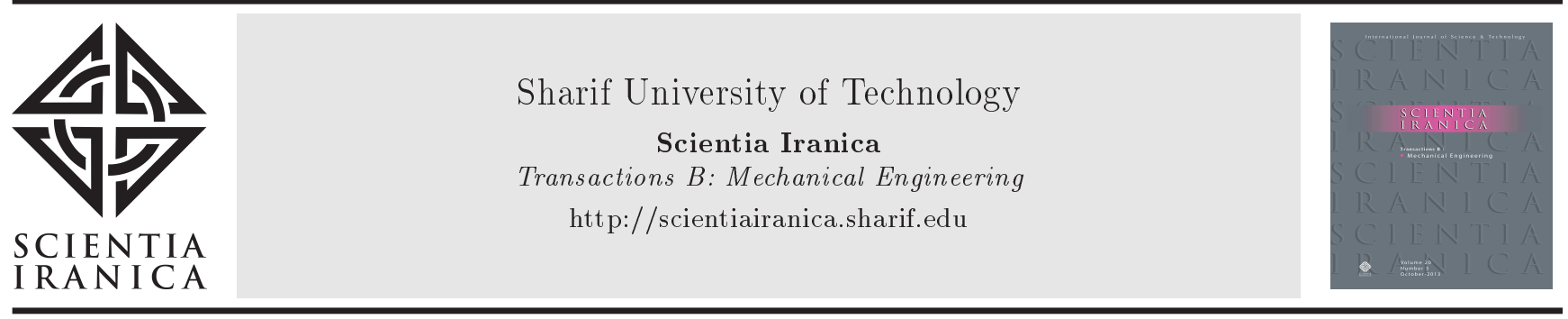

Research Note

\title{
Improvement of dissipative particle dynamics method by taking into account the particle size
}

\author{
S. Yaghoubi ${ }^{\mathrm{a}, *}$, E. Shirani ${ }^{\mathrm{b}}$, and A.R. Pishevar ${ }^{\mathrm{c}}$ \\ a. Department of Mechanical Engineering, Najafabad Branch, Islamic Azad University, Najafabad, Iran. \\ b. Department of Mechanical Engineering, Foolad Institute of Technology, Fooladshahr, Isfahan, P.O. Box 84915651, Iran. \\ c. Center of Excellence in Energy Conversion, Department of Mechanical Engineering, Isfahan University of Technology, Isfahan, \\ P.O. Box 8415683111, Iran.
}

Received 7 August 2016; received in revised form 18 December 2017; accepted 24 September 2018

KEYWORDS
Dissipative particle
dynamics;
Low-dimensional
model;
Low Reynolds
number;
Stokes-Einstein
equation.

\section{Introduction}

In this paper, a flow with low Reynolds number past a single DPD particle as a point center of repulsion is investigated and the minimum length scale is determined in such a way that the hydrodynamic behaviour of flow based on standard DPD formulation is modeled correctly. Many previous studies assumed DPD particles as point centers of repulsion with no intrinsic size. Hence, to prescribe the size of a simulating sphere, a

\footnotetext{
*. Corresponding author. Tel.: +98 3142292884;

Fax: +98 3133912625

E-mail addresses: s.yaghoubi@pmc.iaun.ac.ir

(S. Yaghoubi); eshirani@ictp.it (E. Shirani);

aishe@cc.iut.ac.ir (A.R. Pishevar).
}

structure for frozen DPD particles is proposed. For example, Chen et al. [1] used DPD method to simulate the Stokes flow past a sphere which was constructed by 452 frozen DPD particles. This sphere was surrounded by a flow of free DPD particles. The simulation results were in good agreement with those of the Stokes law. However, in many DPD simulations of colloidal and polymeric solutions, the colloidal particles and polymer beads were represented by individual DPD particles [24], which were proved to be efficient simulations. In this study, the equilibrium radial distribution function, $g(r)$, as a function of distance is plotted and it will be observed that almost no particle can penetrate into this sphere. Then, it will be proved that each of the individual DPD particles interact with other particles as a sphere with non-zero radius.

A linear polymer in Brownian Dynamics (BD) is 
modelled as a chain of beads which are connected to each other with the spring forces. In this method, the size of the spherical beads is determined by the assumption of Stokes law and use of the frictional coefficients. In DPD method, a standard model of linear polymer is a chain of DPD particles which are connected together by the spring forces and immersed in a solution of free DPD particles. Since these models of polymeric solutions are equivalent, it is necessary to introduce an intrinsic size for DPD particles to show that BD beads and DPD particles are equivalent as well. Then, two effective radii in DPD are inferred, which can be calculated independently; Stokes-Einstein radius $\left(R_{S E}\right)$, which is shown in this paper as the radius of a sphere impenetrable around each DPD particle, is calculated by means of the coefficients of self-diffusion and viscosity [5] and the Stokes-Einstein equation. On the other hand, the second radius $\left(R_{S}\right)$ is calculated from the Stokes law in simulating the flow past a single fixed DPD particle. For small Reynolds numbers, it is proved that the two radii approach each other. By considering the Stokes-Einstein radius for DPD particles and representing congruous hydrodynamic behaviour with the analytical Stokes law, it is concluded that each DPD particle has an intrinsic size, which behaves as a solid sphere. In the following sections, the relations governing these radii are described. This conclusion can lead to a considerable decrease in the number of particles in DPD simulating, because each particle represents a bluff body. The effect of number of particles on computational time will be shown quantitatively, which proves that reduction in the number of particles results in more economical simulations.

In this paper, after an introduction to the assumption of DPD point particles, the governing equations of dissipative particle dynamics are briefly described. Then, the flow past individual DPD particles is investigated and by representing the results, the intrinsic size for DPD particles is introduced. Finally, the main points of the paper are summarized in the conclusion.

\section{Governing equations in DPD}

The dissipative particle dynamics method, which was first introduced by Hoogerbrugge and Koelman [6], is a stochastic mesoscopic simulation method [7] and includes particles representing coarse grained molecules that move together in Lagrangian method.

The coarse grained DPD particles interact via three pairwise additive forces, namely repulsion conservative force, $F_{i j}^{C}$, dissipative force, $F_{i j}^{D}$, and random force, $F_{i j}^{R}$, which conserve linear momentum. In this method, it is assumed that these forces vanish beyond a cut-off radius, $r_{c}$. It is assumed that the DPD system consists of $N$ point particles with mass $m_{i}$, position $r_{i}$, and velocity $v_{i}$. The Newton's laws govern the motion of the DPD particles and for a particle $i$, the following relations are satisfied [7]:

$$
\begin{aligned}
& \frac{d r_{i}}{d t}=v_{i} \\
& m_{i} \frac{d v_{i}}{d t}=f_{i}=\sum_{j \neq i}\left(F_{i j}^{C}+F_{i j}^{D}+F_{i j}^{R}\right) .
\end{aligned}
$$

The sum of runs over all other particles resides in a sphere with cut-off radius, $r_{c}$. The three forces are introduced below. The conservation force is a soft-core repulsive force given by [7]:

$$
F_{i j}^{C}= \begin{cases}a_{i j}\left(1-\frac{r_{i j}}{r_{c}}\right) \hat{r}_{i j} & \left(r_{i j}<r_{c}\right) \\ 0 & \left(r_{i j} \geq r_{c}\right)\end{cases}
$$

where, $a_{i j}=\sqrt{a_{i} a_{j}}$; and $a_{i}$ and $a_{j}$ are repulsive parameters for particles $i$ and $j$, respectively. $\vec{r}_{i j}=$ $\vec{r}_{i}-\vec{r}_{j}$ is the distance vector pointed from particle $j$ to $i$ and $r_{i j}=\left|\vec{r}_{i j}\right|$. by [7]:

The dissipative and random forces are given

$$
\begin{aligned}
& F_{i j}^{D}=-\gamma \omega^{D}\left(r_{i j}\right)\left(\hat{r}_{i j} . v_{i j}\right) \hat{r}_{i j}, \\
& F_{i j}^{R}=\sigma \omega^{R}\left(r_{i j}\right) \xi_{i j} \hat{r}_{i j},
\end{aligned}
$$

where, $\gamma$ and $\sigma$ are the amplitudes of dissipative and random forces, respectively. $\omega^{D}$ and $\omega^{R}$ are $r$ dependent weight functions, which are equal to zero for $r>r_{c} . v_{i j}=v_{i}-v_{j}$ is the relative velocity between particles $i$ and $j$, and $\xi_{i j}$ is a normally random variable with zero mean and unit variance $\left(\xi_{i j}=\xi_{j i}\right)$.

Español and Warren [8] showed that in a DPD system with random and dissipative forces, the fluctuation-dissipation theorem should be satisfied as follows:

$$
\omega^{D}(r)=\omega^{R}(r)^{2}, \quad \sigma^{2}=2 \gamma k_{B} T,
$$

where $k_{B}$ is the Boltzmann constant and $T$ is the equilibrium temperature of the system. To estimate the temperature in the simulation, the average kinetic energy for one particle is calculated, which assumes that every particle has the mass 1 DPD unit, and then the coefficient $2 / 3$ is multiplied. Obeying this theorem, DPD produces a thermostat system $[7,8]$. The standard weight function is [7]:

$$
\begin{gathered}
{\left[\omega^{R}(r)\right]=\left\{\begin{array}{l}
\left(1-\frac{r}{r_{c}}\right)\left(r<r_{c}\right) \\
0\left(r \geq r_{c}\right)
\end{array} \quad\right. \text { and }} \\
\omega^{D}(r)=\left[\omega^{R}(r)\right]^{2} .
\end{gathered}
$$

In order to increase the viscosity of DPD fluid, 
Yaghoubi et al. modified the standard weight function and proposed a new formulation as follows [9]:

$\left[\omega^{R}(r)\right]=\left\{\begin{array}{l}\left(1-\frac{r}{r_{c}}\right)^{k}\left(r<r_{c}\right) \\ 0\left(r \geq r_{c}\right)\end{array}\right.$

In this new formulation, if $k=1$, the conventional weight function in standard DPD systems is obtained, but the great defect with the standard weight function is the low Schmidt number for the liquids. Considering $k>1$ leads to increase in viscosity and then, a Schmidt number even more than the modification proposed by Fan et al. [10].

In these simulations, the particle mass, temperature, and interaction range have been chosen as units of mass, energy, and length, respectively, and to evaluate equations of motion, the modified velocityVerlet algorithm is used [7].

\section{Flow past a single DPD particle}

In this section, flow past a single Dissipative Particle Dynamics (DPD) particle as a centre of repulsion is investigated and then, an effective radius is introduced. It will be seen that each DPD particle is surrounded by a spherical space, which is almost impenetrable by other DPD particles. The radius of this sphere is known as Stokes-Einstein radius and the size of the radius depends only on the repulsive coefficient in the conservative force [11]. Calculating the selfdiffusion and viscosity coefficient of the fluid, and using the Stokes-Einstein equation, the radius $R_{S E}$ can be obtained. The Stokes-Einstein equation is given by:

$$
R_{S E}=\frac{k_{B} T}{C D_{\infty} \pi \mu}
$$

where, $D_{\infty}$ is the self-diffusion coefficient of a specific particle, which is subject to Brownian motion in an unbounded domain, and the coefficient $\mu$ is the viscosity of the surrounded fluid. In particle based models such as DPD, the diffusion coefficient can be calculated using displacement data, which is obtained by employing mean square displacement during long term in equilibrium simulations due to the Einstein relation [12]:

$$
D_{\infty}=\lim _{t \rightarrow \infty} \frac{\left\langle|r(t)-r(0)|^{2}\right\rangle}{6 \times t}
$$

where, $<$. $>$ represents the ensemble averaging, and $r(0)$ and $r(t)$ are the particle positions at the initial time and time $t$, respectively. In this paper, the Lees-Edwards periodic boundary conditions are used to determine the viscosity coefficient of the flow [13].
Table 1. DPD simulation parameters.

\begin{tabular}{cccccc}
\hline $\boldsymbol{n}$ & $\boldsymbol{a}$ & $\boldsymbol{\gamma}$ & $\boldsymbol{\sigma}$ & $\boldsymbol{r}_{\boldsymbol{c}}$ & $\boldsymbol{k}_{\boldsymbol{B}} \boldsymbol{T}$ \\
\hline 3 & 25 & 4.5 & 3 & 1 & 1 \\
\hline
\end{tabular}

To show that the Stokes-Einstein sphere surrounding a DPD particle is approximately impenetrable, the equilibrium radial distribution function is required during a simulation time. For this purpose, the initial DPD parameters are listed in Table 1, where $n$ is the number density and $a$ is the conservative force coefficient. Other parameters have been defined previously.

Applying the above parameters, the equilibrium Radial Distribution Function (RDF), $g(r)$, for the DPD particles is plotted in Figure 1. RDF is considered as a function of dimensionless distance, $r / R_{S E}$. In this figure, the area under the curve on the left of the vertical line, $r=R_{S E}$, is negligible relative to the total area under the curve. Therefore, this confirms that the Stokes-Einstein sphere is approximately impenetrable.

\section{Hydrodynamic interactions for individual DPD particles}

In this section, it is investigated whether a single DPD particle immersed in a fluid of identical DPD repulsive particles has an intrinsic size. Then, the minimum length scale is determined such that the hydrodynamic behaviour based on standard DPD formulation is modelled correctly. Before that, DPD particles are assumed as point centres of repulsion with no intrinsic size and then, the size of a simulating sphere is determined by creating a construction of frozen DPD particles. For example, Chen et al. [1] used the DPD method to simulate Stokes flow around a sphere, which was

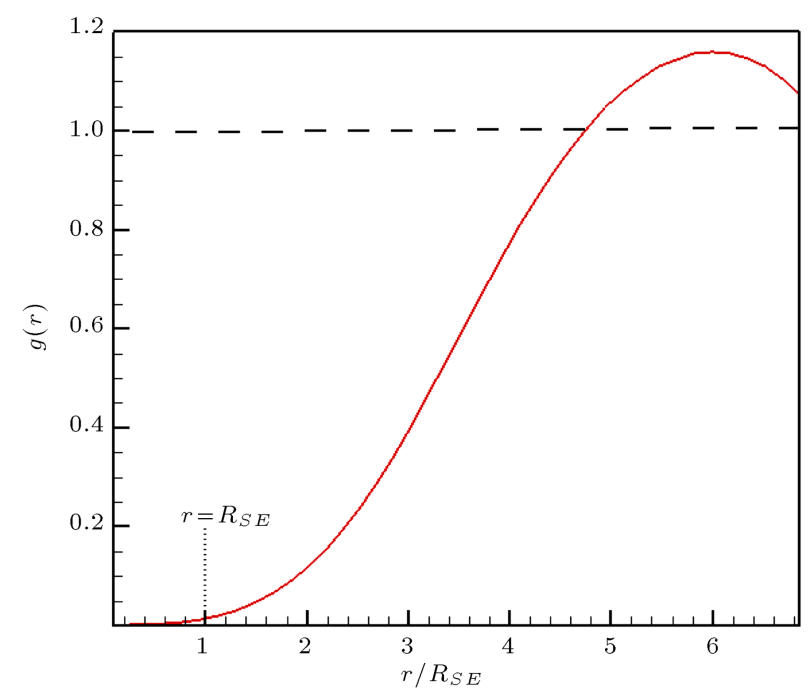

Figure 1. The equilibrium radial distribution function, $g(r)$, as a function of dimensionless distance $r / R_{S E}$; here $R_{S E}=0.144$. 
created by 452 frozen DPD particles and surrounded by a flow of free particles. The results of this simulation agreed well with the Stokes law.

Although each DPD particle is surrounded by a Stokes-Einstein sphere, to prove that it interacts with the other particles as a sphere with intrinsic size, it should be shown that each DPD sphere behaves hydrodynamically correct as well.

For this purpose, a single DPD particle is examined in a simple fluid flow with the uniform velocity, $U_{\infty}$, and viscosity coefficient, $\mu$, in an unbounded domain. It is assumed that the single DPD particle is immersed in this fluid of identical DPD particles and hence, we investigate the hydrodynamic behavior of the single particle. It is already proved that an immersed single DPD particle in a fluid consists of apparently identical DPD point particles and statistically responds as if the surrounding fluid is a continuous system [11]. Moreover, according to Stokes [14], in the analytical solution for a flow with very low Reynolds number past a sphere with the radius $R_{S}$, when the inertia forces are neglected, the drag force on the sphere is given by [14]:

$$
F_{D}=6 \pi \mu U_{\infty} R_{S}
$$

where $\mu$ is the viscosity coefficient and $U_{\infty}$ is the uniform velocity of the flow.

If the sphere behaves hydrodynamically correct in its interactions with the other particles, it should obey Eq. (8) for a flow with very low Reynolds number. To counteract the effect of the drag force on the sphere and fix its position, an external force with the same amount but in the opposite direction is imposed on the central sphere. Then, the position of the sphere is obtained. To instate a suitable condition for the simulation, the amount of the velocity, $U_{\infty}$, must be determined such that $R e<1$.

To obtain some basic parameters, it is required to simulate the fluid flow with only the particles of the fluid. The physical parameters obtained in the initial simulations are summarized in Table 2.

In other simulations, two types of particles are considered; the fluid particles and the central sphere, which is immersed in the fluid. It is assumed that the radius of a single particle is equal to the StokesEinstein radius (i.e. $R_{S}=R_{S E}$ ) and we impose the force $F_{D}$ given by Eq. (9) at the opposite direction of the velocity $U_{\infty}$ on the single DPD sphere. The initial position of the sphere is set at $(0,0,0)$, which is in the centre of the domain, and we investigate the hydrodynamic behaviour of the particle. For simplicity, we

Table 2. Parameters obtained in the initial simulations.

\begin{tabular}{ccccc}
\hline $\boldsymbol{R e}$ & $\boldsymbol{U}_{\boldsymbol{\infty}}$ & $\boldsymbol{R}_{\boldsymbol{S E}}$ & $\boldsymbol{M}$ & $\boldsymbol{D}_{\boldsymbol{\infty}}$ \\
\hline$\sim 0.9$ & 0.5 & $\sim 0.144$ & $\sim 0.24$ & 1.536 \\
\hline
\end{tabular}

consider uniform velocity only in $x$-direction. The $x$ position of the central sphere is plotted as a function of time (Figure 2). According to Figure 2, after some displacements around the origin of the coordinates, the DPD sphere particle soon returns to its original position, $x=0$, and remains approximately in this position. To show that the equilibrium is preserved, the temperature is plotted in Figure 3 for the same period of time. In this figure, it is shown that temperature fluctuation is around $k_{B} T=1$, which guarantees the stability of the system.

Since the position of the particle in a flow with uniform velocity and an external force is constant, it is clear that net force importing on the sphere is nearly zero. It means that the amounts of external force and the drag force in the flow are approximately equal. Therefore, because other parameters such as viscosity

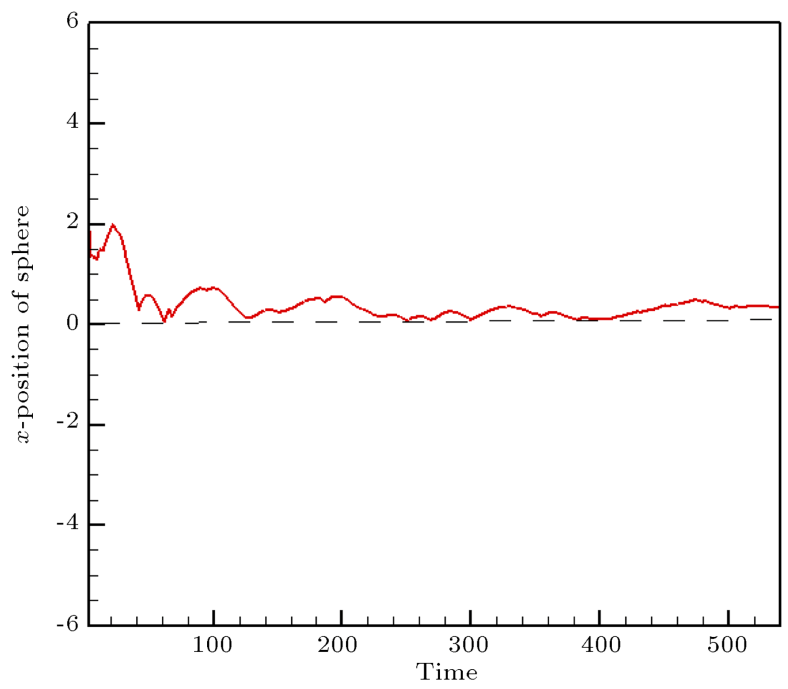

Figure 2. $x$-position of the central sphere as a function of time.



Figure 3. Temperature as a function of time. 
and the velocity of the flow in addition to the drag force are known, the only unknown parameter is the radius of the sphere. The hydrodynamic radius of the particle is equal to Stokes-Einstein size; this fact proves that each DPD particle behaves hydrodynamically as a solid sphere with radius $R_{S E}$.

Accordingly, the number of particles in DPD simulating considerably decreases, because each particle represents a bluff body. The effect of number of particles on computational time is shown below, quantitatively, which proves that the reduction in the number of particles leads to more economical simulations.

\section{Effect of some parameters on Stokes-Einstein radius}

In this study, several simulations have been carried out to investigate the effect of different parameters on Stokes-Einstein radius. These parameters include temperature of the system, particle density, repulsive parameter in conservative force, and different weight functions for dissipative force.

As it is shown in Figure 4, decreasing the repulsive parameter leads to decrease in the Stokes-Einstein radius, because the repulsion power is decreased. Also, as observed in Figure 5, decreasing the temperature of

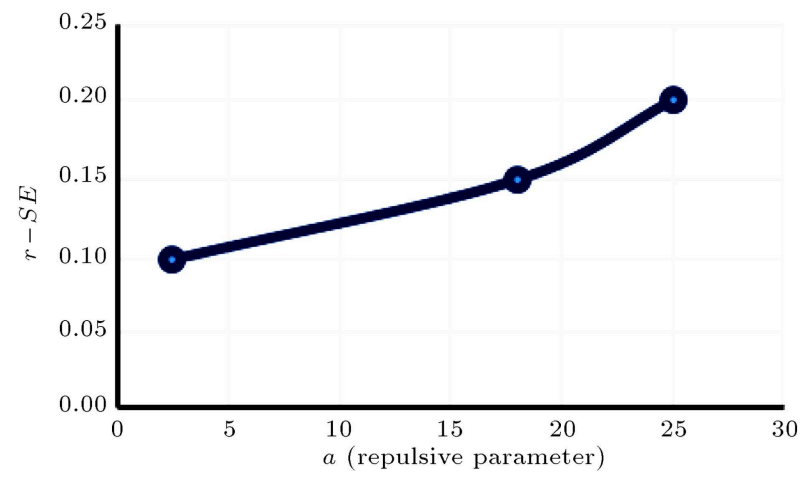

Figure 4. Stokes-Einstein radius with different repulsive parameters.

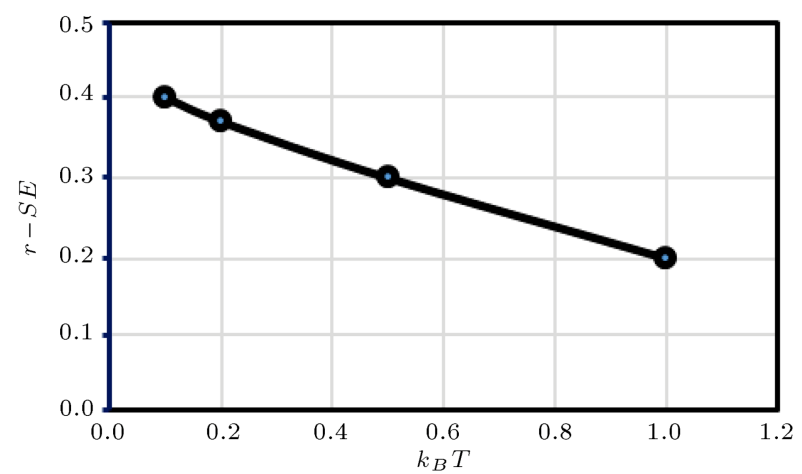

Figure 5. Stokes-Einstein radius versus different temperatures of the system. the system has a reverse effect on the Stokes-Einstein radius and results in larger radius, because the energy level of the other particle is decreased.

In the simulations presented in Figure 6 , it is seen that increasing the density leads to decrease in the Stokes-Einstein radius due to aggregation of the particles.

It is observable in Figures 7 and 8 that when we change the simulated fluid with different weight functions, the Stokes-Einstein radius remains fixed.

Generally, it is concluded that the size of the Stokes-Einstein radius for every particle is only dependent on the repulsive energy level of that particle.

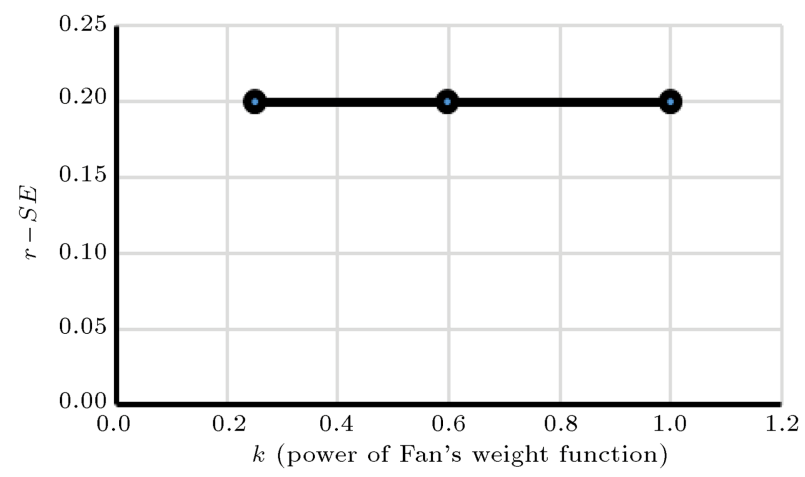

Figure 6. Stokes-Einstein radius versus different particle densities.

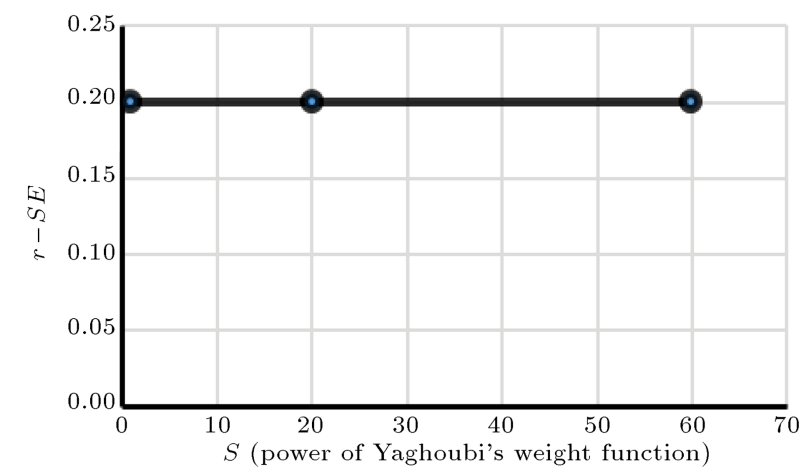

Figure 7. The effect of different weight functions proposed by Fan [10] on Stokes-Einstein radius.

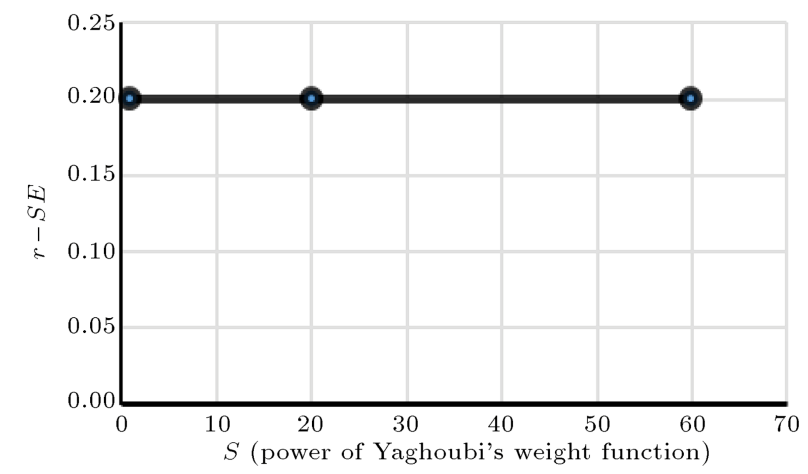

Figure 8. The effect of different weight functions proposed by Yaghoubi [9] on Stokes-Einstein radius. 
With increase in this energy, the size of Stokes-Einstein radius will increase too. Also, changing the fluid with different weight functions cannot affect the StokesEinstein radius.

\section{A more economical DPD method}

One of the most time consuming steps in particlebased methods such as MD and DPD is calculating the forces implemented on each particle. If we consider $N$ particles in the simulating domain, in a very simple view, we must know the positions of the other $N-1$ particles. Hence, the computational cost is in the order of $O\left(N^{2}\right)$. Fortunately, since DPD is a shortrange method, each particle does not need to have interaction with all other $N-1$ particles, but it must interact with particles which are settled in a sphere with the radius $r_{c}$ (cut-off radius) around the target particle. This leads to a considerable decrease in the computational cost by using a suitable searching algorithm such as Verlet neighbour list $[15,16]$ and Cell structure and linked list [15]. It is estimated that the scaling of computational time changes to $O(N)+1 / f O\left(N^{2}\right)$ with Verlet neighbour list, where $f$ is the frequency at which the list is recreated in a time step [15]. Tiwari performed some simulations in a twodimensional domain with periodic boundary conditions and different numbers of particles [17]. The effect of number of particles on computational time per time steps is plotted in Figure 9.

In Figure 9, it is shown that the increase in computational time with the number of particles behaves almost linearly or in a quadratic manner by using or not using Verlet neighbour list, respectively. In this figure, the left curve shows the computational time per

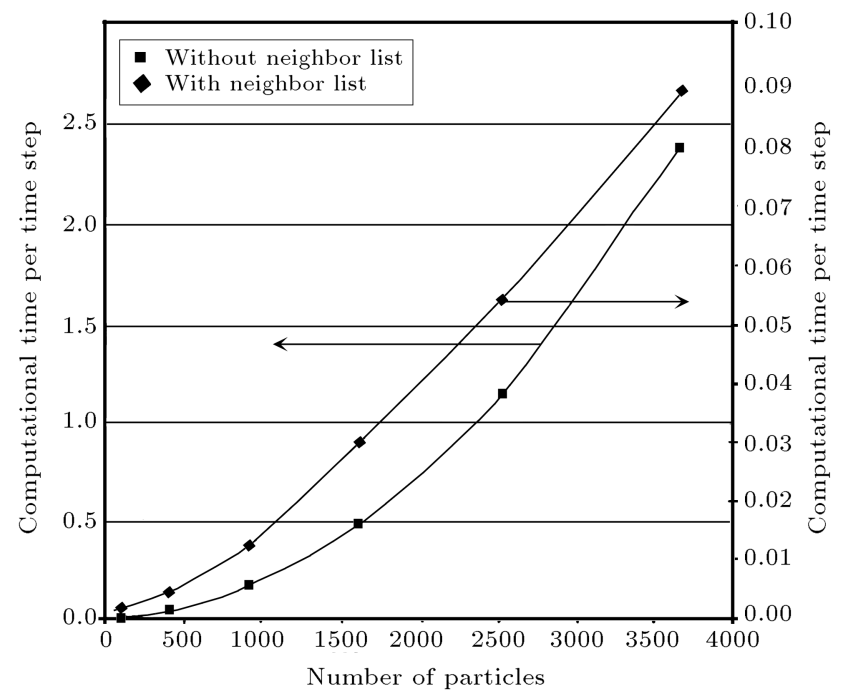

Figure 9. The effect of number of particles on computational time per time steps with and without Verlet neighbor list [17]. time steps with Verlet neighbor list versus the number of particles and the right one shows the same parameter without Verlet neighbor list.

In cell structure and linked list algorithm, the computational domain is assigned to $M \times M \times M$ networks such that the dimensions of each cell, $l=$ $L / M$, should be greater than the cut-off radius. Then, in a two-dimensional system, we have approximately $N_{C}=N / M^{2}$ particles in each cell and in a threedimensional system, $N_{C}=N / M^{3}$ particles. Therefore, using cell structure and linked list algorithm, we only have $9 N N_{C}$ and $27 N N_{C}$ paired particles to search in the 2-D and 3-D systems, respectively [15]. In our simulations, we used this efficient algorithm, which behaved in an almost linear equation with the number of particles. Tiwari performed a three-dimensional simulation in a periodic domain with different numbers of particles, with and without the cell structure and linked list algorithm [17]. The effect of the number of particles on computational time per time steps is plotted in Figure 10. In this figure, the left curve shows the computational time per time steps with linked list algorithm versus the number of particles and the right one shows the same parameter without linked list algorithm.

Using particles with intrinsic size results in reduction in the required number of particles for simulations. In this section, the effect of the number of particles on computational time is shown quantitatively, which proves that reduction in the number of particles leads to more economical simulations.

Moreover, in Low-Dimensional (LD) model [18], which is established based on DPD [6,7] and FPM [19] methods, the particles radius is considered essential. In this method, the deficiency recognized in standard

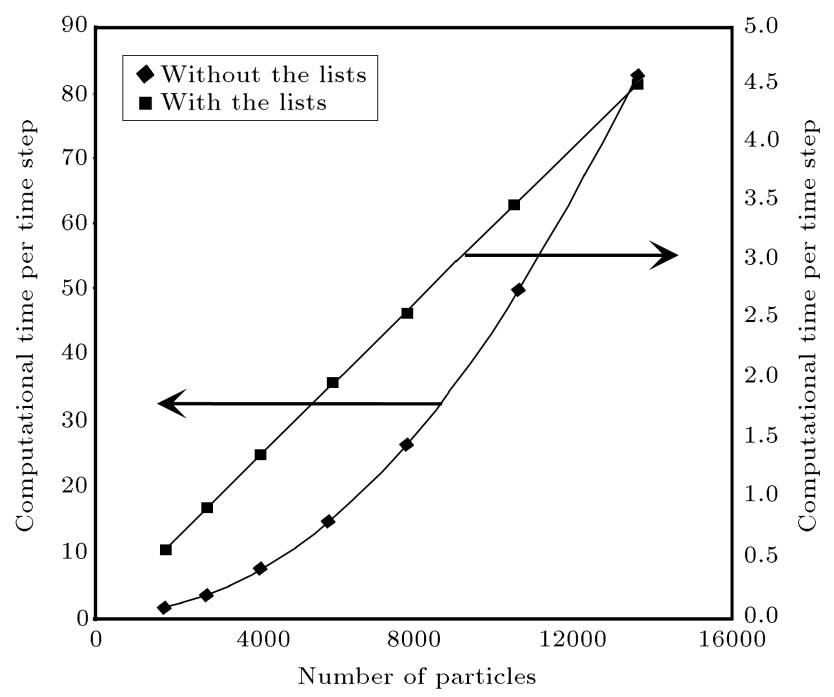

Figure 10. The effect of number of particles on computational time per time steps with and without the cell structure and linked list algorithm [17]. 
DPD is solved. Also, because of the existence of the particles with radius, this approach is able to interpret the essential mechanical and rheological properties correctly and economically [18]. In fact, the suspended particles in a flow have both translational and rotational movements, whereas in standard DPD, a single particle perceives only central forces. Thus, in the standard DPD simulation, the non-central shear forces between particles, and the angular momentum and torque for each single DPD particle are ignored. Espanol et al. [19-21] recognized this defect in the standard DPD and proposed a more complicated Fluid Particle Model (FPM) [19]. In this method, the noncentral shear components, angular momentum, and torque for each single particle are considered. Based on the improvement represented by Espanol [19], a set of new formulations for DPD technique were derived by $\mathrm{Pan}$ et al. [22], which had the implementation and computational simplicity of the standard DPD method and yielded correct hydrodynamics in flows around bluff bodies represented by a single particle. The LD method is a generalized version of DPD based on this new formulation [20] and seeks to resolve the DPD deficiency. This approach can be used with confidence in studying the properties of suspended particles in a fluid phase, including the transport of macromolecules, colloids, biomolecules such as DNA, and blood cells such as Red Blood Cells (RBCs) [18]. The peculiarities of these systems are often based on their mesoscale structures. Mesoscale properties are the structural features with the scale between the atomistic (microscopic) and continuum (macroscopic) levels. These structural features endue unique and interesting peculiarities to the complex fluids. For instance, LD model is able to interpret the essential mechanical and rheological properties of the red cells in blood flow correctly and economically [18].

\section{Conclusions}

In this paper, it is shown that a single DPD particle immersed in a fluid consisting of identical repulsive DPD particles has an intrinsic size. A minimum length scale was determined such that the hydrodynamic behaviour based on the standard DPD formulation was modelled correctly. Many previous studies assumed DPD particles as point centres of repulsion with no intrinsic size and hence, to prescribe the size of a simulating sphere, created a structure of frozen DPD particles. For example, Chen et al. [1] used DPD method to simulate Stokes flow past a sphere which was constructed by 452 frozen DPD particles. Moreover, in this paper, two effective radii in DPD were introduced, which could be calculated independently: the StokesEinstein radius $\left(R_{S E}\right)$ and the hydrodynamics radius $\left(R_{S}\right)$. It was proved that each of the DPD particles were surrounded by a spherical space, which was approximately impenetrable for the other DPD particles. The radius of this sphere was known as Stokes-Einstein and its size depended only on the repulsive coefficient in the conservative force. The hydrodynamics radius was calculated from the Stokes law in simulating the flow past a single fixed DPD particle. In the limit, where the Reynolds number was small, the two radii approached each other.

Based on the fact that the Stokes-Einstein radius was equivalent to hydrodynamics radius of the DPD particle in low Reynolds number flows, it was concluded that, in spite of the previous studies that assumed DPD particles as point centres of repulsion with no intrinsic size, each of the individual DPD particles interacted with the other particles as a solid sphere with non-zero radius.

Using particles with intrinsic size led to the reduction in the required number of particles for simulations. The effect of the number of particles on computational time was shown quantitatively, which proved that reduction in the number of particles would result in more economical simulations.

Contemplating the radius of the particles is necessary for the Low-Dimensional (LD) model, which is derived based on DPD [6,7] and FPM [19] methods. For instance, the LD model of the red blood cells is able to interpret the essential mechanical and rheological properties correctly and economically [18].

\section{References}

1. Chen, S., Phan-Thien, N., Khoo, B.C., and Fan, X.J. "Flow around spheres by dissipative particle dynamics", Physics of Fluids, 18(10), 103605 (2006).

2. Pryamitsyn, V. and Ganesan, V. "A coarse-grained explicit solvent simulation of rheology of colloidal suspensions", Journal of Chemical Physics, 122(10), 104906 (2005).

3. Symeonidis, V., Caswell, B., and Karniadakis, G.E. "Dissipative particle dynamics simulations of polymer chains: Scaling laws and shearing response compared to DNA experiments", Physical Review Letters, 95, 076001 (2005).

4. Yaghoubi, S., Pishevar, A.R., Saidi, M.S., and Shirani, E. "Modeling self-assembly of the surfactants into biological bilayer membranes with special chemical structures using dissipative particle dynamics method", Scientia Iranica, 23(3), pp. 942-950 (2016).

5. Kumar, A., Asako, Y., Abu-nada, E., Krafczyk, M., and Faghri, M. "From dissipative particle dynamics to physical scales: A coarse-graining study for water flow in microchannel", Microfluid Nanofluid Journal, 7, pp. 467-477 (2009).

6. Hoogerbrugge, P.J. and Koelman, J.M.V.A. "Simulating microscopic hydrodynamic phenomena with 
dissipative particle dynamics", Europhys. Lett., 19(3), pp. 155-160 (1992).

7. Groot, R.D. and Warren, P.B. "Dissipative particle dynamics: Bridging the gap between atomistic and mesoscopic simulation", Journal of Chemical Physics, 107(11), pp. 4423-4435 (1997).

8. Espanol, P. and Warren, P.B. "Statistical mechanics of dissipative particle dynamic", Europhysics Letters, 30(4), pp. 191-196 (1995).

9. Yaghoubi, S., Shirani, E., Pishevar, A.R., and Afshar, Y. "New modified weight function for the dissipative force in the DPD method to increase the Schmidt number", Europhysics Letters, 110, 24002 (2015).

10. Fan, X.J., Phan-Thien, N., Chen, S., Wu, X.H., and $\mathrm{Ng}, \mathrm{T} . \mathrm{Y}$. "Simulating flow of DNA suspension using dissipative particle dynamics", Physics of Fluids, 18(6), p. 063102 (2006).

11. Pan, W., Fedosov, D.A., Karniadakis, G.E., and Caswell, B. "Hydrodynamic interactions for single dissipative-particle-dynamics particles and their clusters and filaments", Physical Review E, 78(4), 046706 (2008).

12. Frenkel, D. and Smit, B., Understanding Molecular Simulation from Algorithms to Applications, Academic Press, California (2002).

13. Lees, A.W. and Edwards, S.F. "The computer study of transport processes under extreme conditions", $J$. of Phys. C: Solid State Physics, 5, 1921 (1972).

14. Fax, R.W. and McDonald, A.T., Introduction to Fluid Mechanics, Wiley Publications, fourth edition (1978).

15. Allen, M.P. and Tildesley, D.J., Computer Simulation of Liquids, Oxford Science Publications, Oxford (1987).

16. Verlet, L. "Computer experiment on classical fluids I. Thermodynamical properties of Lennard-Jones molecules", Physical Review, 159, pp. 98-103 (1967).

17. Tiwari, A., "Dissipative particle dynamics model for two phase flows", Ph.D. Thesis, University of Purdue (2006).
18. Pan, W., "Single particle DPD: Algorithms and applications", Ph.D. Thesis, University of brown, (2010).

19. Espanol, P. "Fluid particle model", Physical Review E, 57(3), pp. 2930-2948 (1998).

20. Espanol, P. "Fluid particle dynamics: A synthesis of dissipative particle dynamics and smoothed particle dynamics", Europhysics Letters, 39(6), pp. 605-610 (1997).

21. Espanol, P. and Revenga, M. "Smoothed dissipative particle dynamics", Physical Review E, 67, 026705 (2003).

22. Pan, W., Pivkin, I.V., and Karniadakis, G.E. "Singleparticle hydrodynamics in dpd: A new formulation", Europhysics Letters, 84(1), 10012 (2008).

\section{Biographies}

Somaye Yaghoubi is Associated Professor of Mechanical Engineering at Najafabad Branch, Islamic Azad University, Iran. She obtained her BS, MS, and $\mathrm{PhD}$ degrees from Isfahan University of Technology, Iran. Her research interests are biofluid simulation, modeling of particle transport, CFD simulations, and energy conversion.

Ebrahim Shirani is Professor of Mechanical Engineering at Isfahan University of Technology, Iran, and at Foolad Institute of Technology, Fooladshahr, Isfahan, Iran. He is Editor-in-Chief of the Journal of Applied Fluid Mechanics.

Ahmad Reza Pishevar is Professor of Mechanical Engineering at Isfahan University of Technology, Iran. He is Editor-in-Chief of the Journal of Computational Methods in Engineering. His research interests include compressible unsteady flow, multiphase flows, and CFD simulations. 